\title{
LINEAR AND NONLINEAR SEMIDEFINITE PROGRAMMING
}

\author{
Walter Gómez Bofill* and Juan A. Gómez
}

Received November 7, 2013 / Accepted January 19, 2014

\begin{abstract}
This paper provides a short introduction to optimization problems with semidefinite constraints. Basic duality and optimality conditions are presented. For linear semidefinite programming some advances by dealing with degeneracy and the semidefinite facial reduction are discussed. Two relatively recent areas of application are presented. Finally a short overview of relevant literature on algorithmic approaches for efficiently solving linear and nonlinear semidefinite programming is provided.
\end{abstract}

Keywords: Semidefinite programming, nonlinear semidefinite programming, Euclidean completion matrices.

\section{INTRODUCTION}

Semidefinite programming (SDP) is one of the most vigorous and fruitful research topics in optimization the last two decades. The intense activity on this area has involved researchers with quite different mathematical background reaching from nonlinear programming to semialgebraic geometry. This tremendous success of the semidefinite programming model can be explained by many factors. First, the existence of polynomial algorithms with efficient implementations that made the SDP model tractable in many situations. Second, the endless list of quite different and important fields of applications, where SDP has proved to be a useful tool. Third, the beauty and depth of the underlying theory, that links in a natural way different and usually unrelated areas of mathematics.

There are many and excellent survey papers [138, 137, 133, 58, 38, 92, 95, 87] and books $[101,28,37,18,16,29,86,19]$ covering the Semidefinite Programming model with algorithms and special applications. The previous list of references is by no means complete, but only a short overview on a incresing and large set of items. A special mention in the literature on Semidefinite Programming deserves the Handbook of Semidefinite Programming [141] edited by H. Wolkowicz, R. Saigal and L. Vandenberghe in 2000, that covered the principal results on

\footnotetext{
*Corresponding author.

Departamento de Ingeniería Matemática, Universidad de la Frontera, Temuco, Chile.

E-mail: walter.gomez@ufrontera.cl
} 
the area during the 1990's. After the publication of the mentioned Handbook the research activity in Semidefinite Programming continued growing and new areas of development were added. In particular the interaction with algebraic geometry and the exploration of the close relationship between semidefinite matrices and polynomial optimization gave rise to important new results and to an even higher level of research activity. As recent as 2012 it appeared a new Handbook on Semidefinite, Conic and Polynomial Optimization edited by M.F. Anjos and J.B. Lasserre [9]. This new Handbook provides in 30 chapters a complete update of the research activity on the area in the last decade.

Our main intention in this short review is to motivate researchers to become involved in this amazing area of research. We focus on readers with a basic background in continuous Optimization, but without a previous knowledge in Semidefinite Programming. Our goal is to provide a simple access to some of the basic concepts and results in the area and to illustrate the potential of this model by presenting some selected applications. A short overview on the theoretical and algorithmic results in the case of nonlinear semidefinite programming is also given. We suggest to readers interested in a more detailed exposition of the semidefinite model to revise the above mentioned Handbooks and the references therein.

The paper is divided into two sections. The first one is devoted to the (linear) Semidefinite Programming and the second one to the case of nonlinear Semidefinite Programming.

\section{THE LINEAR SEMIDEFINITE PROGRAMMING}

The linear semidefinite programming can be intended as linear programming over the cone of positive semidefinite matrices. In order to formulate the problem in details let us fix some notations. In the sequel we denote with $\mathbb{S}^{m}$ the linear space of $m \times m$ real symmetric matrices equipped with the inner product

$$
\langle A, B\rangle:=\operatorname{Tr}(A B)=\sum_{i, j=1}^{m} A_{i j} B_{i j}
$$

where $A=\left(A_{i j}\right), B=\left(B_{i j}\right) \in \mathbb{S}^{m}$.

On this linear space we consider the positive semidefinite order, i.e. $A \succeq B$ iff $A-B$ is a positive semidefinite matrix. The order relations $\succ$ and $\prec, \preceq$ are defined similarly.

The primal semidefinite programming problem is then defined as follows:

$$
\begin{array}{lll} 
& \min & \langle C, X\rangle \\
(S D P-P) & & \mathcal{A} X=b \\
& X \succeq 0
\end{array}
$$

where $C \in \mathbb{S}^{m}, b \in \mathbb{R}^{n}$ are given data, and $\mathcal{A}: \mathbb{S}^{m} \rightarrow \mathbb{R}^{n}$ is a linear operator. In general $\mathcal{A}$ is written as

$$
\mathcal{A} X=\left(\begin{array}{c}
\left\langle A_{1}, X\right\rangle \\
\vdots \\
\left\langle A_{n}, X\right\rangle
\end{array}\right)
$$


where $A_{1}, \ldots, A_{n} \in \mathbb{S}^{m}$ are also data of the problem.

Let us denote the set of positive semidefinite matrices as follows

$$
\mathbb{S}_{+}^{m}=\left\{A \in \mathbb{S}^{m} \mid A \succeq 0\right\}
$$

The set $\mathbb{S}_{+}^{m}$ is a full-dimensional, convex closed pointed cone, such that (SDP-P) is a convex problem. Its boundary is the set of semidefinite matrices having at least a zero eigenvalues and its interior is the cone of positive definite matrices. The cone $\mathbb{S}_{+}^{m}$ is also self-dual, i.e. its polar cone

$$
\left(\mathbb{S}_{+}^{m}\right)^{*}=\left\{B \in \mathbb{S}^{m} \mid\langle A, B\rangle \geq 0, \forall A \in \mathbb{S}_{+}^{m}\right\}
$$

coincides with $\mathbb{S}_{+}^{m}$. This property allows to calculate the Lagrange dual of the (SDP). Let the optimal value of (SDP-P) be denoted as follows.

$$
\begin{aligned}
p^{*} & =\inf _{X \in \mathbb{S}_{+}^{m}} \sup _{y \in \mathbb{R}^{n}}\left\{\langle C, X\rangle-\sum_{i=1}^{n} y_{i}\left(\left\langle A_{i}, X\right\rangle-b_{i}\right)\right\} \\
& \left.=\inf _{X \in \mathbb{S}_{+}^{m}} \sup _{y \in \mathbb{R}^{n}}\left\{\left\langle C-\sum_{i=1}^{n} y_{i} A_{i}, X\right\rangle+b^{T} y\right)\right\}
\end{aligned}
$$

Interchanging "sup" and "inf" we obtain the dual with corresponding optimal value $d^{*}$.

$$
d^{*}=\sup _{y \in \mathbb{R}^{n}} \inf _{X \in \mathbb{S}_{+}^{m}}\left\{\langle C, X\rangle-\sum_{i=1}^{n} y_{i}\left(\left\langle A_{i}, X\right\rangle-b_{i}\right)\right\}
$$

Taking into account the selfduality of $\mathbb{S}_{+}^{m}$ the following expression is then obtained:

$$
\left.\inf _{X \in \mathbb{S}_{+}^{m}}\left\{\left\langle C-\sum_{i=1}^{n} y_{i} A_{i}, X\right\rangle+b^{T} y\right)\right\}= \begin{cases}b^{T} y, & C-\sum_{i=1}^{n} y_{i} A_{i} \in \mathbb{S}_{+}^{m} \\ -\infty, & \text { else }\end{cases}
$$

Consequently the dual problem to (SDP-P) can be written as:

$$
\begin{array}{lll} 
& \max & b^{T} y \\
(S D P-D) \quad & \mathcal{A}^{*} y+Z=C \\
& Z \succeq 0
\end{array}
$$

where $\mathcal{A}^{*}: \mathbb{R}^{n} \rightarrow \mathbb{S}^{m}$ denotes the adjoint operator of $\mathcal{A}$ defined as

$$
\mathcal{A}^{*} y=\sum_{i=1}^{n} y_{i} A_{i}
$$

This pair of primal and dual problems has the same structure of primal-dual problems in linear programming with the standard form. The only difference is that the cone defining the inequalities is now $\mathbb{S}_{+}^{m}$ instead of the cone of vectors with nonnegative components. This SDP model contains as special cases many other optimization problems as linear programming, convex quadratic 
programming, second order cone programming, etc. A rich list of semidefinite representable sets and problems can be found, for instance, in [18].

Many of the theoretical and algorithmic results from LP can be carried over to the SDP case. A first trivial one is the weak duality, since from the feasibility of $X$ for (SDP-P) and $(y, Z)$ for (SDP-D) it follows that

$$
\langle C, X\rangle-b^{T} y=\left\langle\mathcal{A}^{*} y, X\right\rangle-b^{T} y+\langle Z, X\rangle=y^{T} \mathcal{A} X-b^{T} y+\langle Z, X\rangle=\langle Z, X\rangle \geq 0
$$

where the last inequality is again a consequence of the self duality of $\mathbb{S}_{+}^{m}$.

From the above weak duality results it follows straightforwardly that the Karush-Kuhn-Tucker system (2) provides sufficient optimality conditions for the pair (SDP-P) and (SDP-D).

$$
\begin{aligned}
\mathcal{A} X & =b \\
\mathcal{A}^{*} y+Z & =C \\
\langle Z, X\rangle & =0 \\
Z, X & \succeq 0
\end{aligned}
$$

Since the cone $\mathbb{S}_{+}^{m}$ is nonpolyhedral, the SDP is a convex but nonlinear optimization problem. In consequence not every nice duality properties of LP can be extended to SDP. For instance, there are solvable primal and dual pairs having a strictly positive duality gap. There are also primal dual problems with zero duality gap that are not both solvable, see for instance [141]. Such examples are impossible in LP and imply also that the above conditions (2) are not necessary for optimality.

The usual way to state strong duality results in the SDP setting is to require the Slater's Constraint Qualification (Slater-CQ). This can be intended for SDP problems as strict feasibility. For (SDPP) it means the existence of a positive definite feasible point $X \succ 0$. Analogously for (SDP-D) it means the existence of a feasible solution $(y, Z)$ with $Z \succ 0$. Under the strict feasibility assumptions the following strong duality results are known.

Theorem 2.1. Let consider the dual problems $(S D P-P)$ and $(S D P-D)$ with optimal values

$$
\begin{aligned}
& p^{*}=\inf \left\{\langle C, X\rangle \mid \mathcal{A} X=b, X \in \mathbb{S}_{+}^{m}\right\} \\
& d^{*}=\sup \left\{b^{T} x \mid \mathcal{A}^{*} y+Z=C, Z \in \mathbb{S}_{+}^{m}, y \in \mathbb{R}^{n}\right\}
\end{aligned}
$$

1. If the problem (SDP-P) is strictly feasible and $p^{*}$ is finite, then $p^{*}=d^{*}$ and the dual optimal value $d^{*}$ is attained.

2. If the problem $(S D P-D)$ is strictly feasible and $d^{*}$ is finite, then $p^{*}=d^{*}$ and the primal optimal value $p^{*}$ is attained.

3. If both problems (SPD-P) and (SDP-D) are strictly feasible, then $p^{*}=d^{*}$ and both optimal values are attained. 
In particular the last strong duality result implies that under the Slater-CQ the above conditions (2) actually characterize primal-dual optimal points. The complementarity condition $\langle Z, X\rangle=0$ in (2) can be equivalently replaced, see e.g. [37], by the usual matrix multiplication, such that the optimality conditions take the form:

$$
\begin{aligned}
\mathcal{A} X & =b \\
\mathcal{A}^{*} y+Z & =C \\
Z X & =0 \\
Z, X & \succeq 0
\end{aligned}
$$

The notion of strict complementarity and degeneracy can be extended to the SDP setting. For instance, strict complementarity means $X+Z \succ 0$, see e.g. [7, 22]. However, not all the properties related to these concepts in LP can be carried over to SDP. In particular the classical theorem of Goldman and Tucker [54] on the existence of primal-dual strict complementarity solutions does not hold for SDP, see for instance [37] also for a discussion on maximal complementary solutions. In fact, the study of nondegeneracy in SDP requires a deeper analysis of the geometry of the semidefinite cone [114].

The Slater-CQ is a generic condition [7, 44]. It is also a crucial condition for the stability of most of the efficient solutions methods for SDP. The Slater-CQ holds also in many applications, for instance for the basic SDP relaxations of the max-cut problem. More details on SDP relaxations of the max-cut problem and other combinatorial problems can be found in [53, 140, 87, 40, 39]

There are however many SDP instances arising for instance also by relaxations of hard combinatorial problems where the Slater-CQ is not fulfilled, see for example [146, 82, 82, 83, 32]. A prevailing approach to get equivalent instances satisfying the Slater-CQ is the skew-symmetric embedding, see [41,37]. This technique uses homogenization of the problem and increases the number of variables.

Another general approach to deal with the lack of strict feasibility bases on the so called facial reduction and extended duals [24, 25, 26, 122, 123, 135, 115]. Let us discuss this second approach, since it uses geometric properties of the semidefinite cone and provides in general smaller regularized problems.

A cone $F \subseteq \mathbb{S}^{m}$ is a face of $\mathbb{S}_{+}^{m}$, denoted by $F \unlhd \mathbb{S}_{+}^{m}$ (and $F \triangleleft \mathbb{S}_{+}^{m}$ in case $F \neq \mathbb{S}_{+}^{m}$ ), if

$$
A, B \in \mathbb{S}_{+}^{m},(A+B) \in F \Rightarrow A, B \in F .
$$

Obviously $\{0\} \triangleleft \mathbb{S}_{+}^{m}$. If $\{0\} \neq F \triangleleft \mathbb{S}_{+}^{m}$, then $F$ is called a proper face of $\mathbb{S}_{+}^{m}$. If $F \unlhd \mathbb{S}_{+}^{m}$ the conjugate or complementary face of $F$, denoted by $F^{c}$, is defined as $F^{c}=F^{\perp} \cap \mathbb{S}_{+}^{m}$. Moreover, if $A$ is in the relative interior of a face $F \unlhd \mathbb{S}_{+}^{m}$, then $F^{c}=\{A\}^{\perp} \cap \mathbb{S}_{+}^{m}$. Detailed results on the facial structure of $\mathbb{S}_{+}^{m}$ can be found, for instance in [113]. The following characterization of the faces of the semidefinite cone is known.

Theorem 2.2. A cone $F \neq\{0\}$ is a face of $\mathbb{S}_{+}^{m}$ if and only if

$$
F=\left\{A \in \mathbb{S}^{m} \mid A=P W P^{T}, W \in \mathbb{S}_{+}^{k}\right\}
$$


for some $k \in\{1, \ldots, m\}$, and $P \in \mathbb{R}^{m \times k}$ with rank $k$.

Let us consider the dual set of feasible slack variables $\mathcal{F}_{D}=\left\{Z \in \mathbb{S}_{+}^{m} \mid Z=C-\mathcal{A}^{*} y\right\}$. The corresponding minimal face is defined as

$$
f_{D}=\operatorname{face}\left(\mathcal{F}_{D}\right)=\bigcap\left\{H \unlhd \mathbb{S}_{+}^{m} \mid \mathcal{F}_{D} \subset H\right\}
$$

The face $\operatorname{face}\left(\mathcal{F}_{D}\right)$ is the smallest face of $\mathbb{S}_{+}^{m}$ containing $\mathcal{F}_{D}$. Using the order $\preceq_{f_{D}}$ derived from the cone $f_{D}$, i.e. $A \preceq f_{D} B \Leftrightarrow B-A \in f_{D}$, a regularized dual problem can be defined.

$$
\left(S D P_{\text {reg }}-D\right) \quad d_{\text {reg }}^{*}=\sup \left\{b^{T} y \mid \mathcal{A}^{*} y \preceq f_{D} C\right\}
$$

The above regularized problem is equivalent to $(S D P-D)$, see $[24,25]$, in the sense that the feasible set remains the same

$$
\mathcal{A}^{*} y \preceq f_{D} C \Leftrightarrow \mathcal{A}^{*} y \preceq C
$$

The Lagrangian dual of $\left(S D P_{r e g}-D\right)$ can be easily calculated as

$$
\left(S D P_{\text {reg }}-P\right) \quad p_{\text {reg }}^{*}=\inf \left\{\langle C, X\rangle \mid \mathcal{A} X=b, X \succeq_{f_{D}^{*}} 0\right\}
$$

where the dual cone is given by

$$
f_{D}^{*}=\left\{Y \in \mathbb{S}^{m} \mid\langle Y, X\rangle \geq 0, \forall Y \succeq f_{D} 0\right\}
$$

The following theorem [24] provides then a strong stability result for the regularized dual problem.

Theorem 2.3. If the original problem optimal value $d^{*}$ in (1) is finite, then $d^{*}=d_{\text {reg }}^{*}=p_{\text {reg }}^{*}$ and the optimal value $p_{\text {reg }}^{*}$ is attained.

Recently a backward stable preprocessing algorithm has been developed that bases on the above semidefnite facial reduction and can provide equivalent regular reformulations to problems without the Slater-CQ, e.g. [34]. In particular the following auxiliary problem is considered

$$
\begin{aligned}
\min _{\delta, D} & \delta \\
& \left\|\left[\begin{array}{c}
\mathcal{A} D \\
\langle C, X\rangle
\end{array}\right]\right\|_{2} \leq \delta \\
& \left\langle\frac{1}{\sqrt{n}} I, D\right\rangle=1 \\
& D \succeq 0
\end{aligned}
$$

This auxiliary problem can be written as a Semidefinite Programming, where in particular the first constraint is a second order cone constraint that can be also written as a semidefinite one, see for instance [18]. The problem (6) and its dual satisfy the Slater-CQ [34]. Consequently, using interior point methods an optimal solution $\left(\delta^{*}, D^{*}\right)$ in the relative interior of the optimal solution set can be obtained. In the most interesting case we get a description of the minimal face as

$$
f_{P}=Q \mathbb{S}_{+}^{\bar{m}} Q^{T}
$$


for some matrix $Q \in \mathbb{R}^{m \times \bar{m}}$ with $Q^{T} Q=I_{\bar{m}}$ and $\bar{m}<m$. A regularized reduction is then obtained, since the original semidefinite program (SDP-D) can be equivalently formulated as reduced problem satisfaying the Slater-CQ, see [34].

Theorem 2.4. Let the feasible set $\mathcal{F}_{D}$ be nonempty and $\left(\delta^{*}, D^{*}\right)$ be a solution of the auxiliary problem (6). If $\delta^{*}=0$ and

$$
D^{*}=\left[\begin{array}{ll}
P & Q
\end{array}\right]\left[\begin{array}{cc}
\Delta_{+} & 0 \\
0 & 0
\end{array}\right]\left[\begin{array}{l}
P^{T} \\
Q^{T}
\end{array}\right],
$$

where $[P Q]$ is orthogonal, $Q \in \mathbb{R}^{m \times \bar{m}}$ and $\Delta_{+} \succ 0$, then (SDP-D) is equivalent to the following problem

$$
\begin{array}{ll}
\sup & b^{T} y \\
& P^{T}\left(\mathcal{A}^{*} y-C\right) P=0 \\
& Q^{T}\left(\mathcal{A}^{*} y-C\right) P=0 \\
& Q^{T}\left(\mathcal{A}^{*} y-C\right) Q \preceq 0
\end{array}
$$

The above remarkable result shows a way to identify hidden linear equality constraints into degenerated SDP problems. This procedure is well established in linear programming as part of general preprocessing steps, but it is not usual in nonlinear problems (as the SDP model). The facial reduction procedure to obtain regularized and reduced problems have been sucessfully used in different application in order to take advantage of degeneracy, see for instance [82, 13, $15,14,32]$.

In the seminal work [101] it was shown that the function $\log (\operatorname{det}(X))$ is a self-concordant barrier function. As a consequence SDP instances can be solved in polynomial time using a sequence of barrier subproblems. In [6] another fundamental approach based on the potential function methods was presented. Strong numerical result were also early reported for min-max eigenvalue problems [60, 63].

There is a long list of quite different algorithmic approaches for solving the SDP problem, see for instance $[6,18,101,103,66,72,99,59,75,95,96,31,30,81,55,117,147,116]$, among many others. The previous list is by far incomplete and we do not intend to describe here all the diverse ideas to deal with the efficient solution of semidefinite programming. Instead we point out to the excellent surveys in the algorithmic sections of the already mentioned Handbooks $[141,9]$. There are many software tools available for solving general SDP problems, for instance SeDumi [130], SDPNAL [147], SDPT3 [136, 134], SDPA [145, 144] and PENNON [71, 75, 77], among others. A useful tool for modelling with SDP and for using the existing SDP-software is the program YALMIP [93]. For a detailed survey about software tools for SDP see [94]. There are also some available implementations of solvers for particular structured SDP problems, we just mention in this direction GloptiPoly $[62,61]$ for the so called generalized problem of moments $[84,85,86]$ and SOSTOOL $[121,120]$ for solving sum of squares optimization programs $[110,111,19]$. 
We present in the rest of this section two selected areas of application of the semidefinite programming model.

\subsection{Polynomial Lyapunov functions}

One example of an important mathematical problem is the search of general methods to prove that a real $n$-variable polynomial $p(\mathbf{x}) \in \mathbb{R}[\mathbf{x}]$ is nonnegative, i.e.

$$
p(\mathbf{x}) \geq 0, \forall \mathbf{x} \in \mathbb{R}^{n} .
$$

This problem is connected with the famous $17^{\text {th }}$ Hilbert problem. It is NP-hard and has not a general computable solution. On the other hand, it is known that a single polynomial $p$ is a nonnegative polynomial if and only if it can be written as a sum of squares of rational functions, and so, clearing denominators $h p=f$ for some sum of squares polynomials $h, f$ ([11],[42]).

Hence, the general question can be transformed into a more restricted but more accesible question: When a given polynomial can be decomposed in a sum of squares of other polynomials? This last question can be answered in a computable way using SDP and the idea was first appeared in [27]. See also [118] for an extense survey of this and other methods to tackle the problem.

If $n=1$ the ring $\mathbb{R}[x]$ of real polynomials of a single variable has the fundamental property that every nonnegative polynomial $p \in \mathbb{R}[x]$ is a sum of squares of some other polynomials. But for $n>1$ not every nonnegative polinomial can be decomposed in a sum of square, but when it does the question is strongly related with a SDP problem. The following definitions and results can be seen in [86].

Let $\mathbb{R}[\mathbf{x}]$ denote the ring of real polynomials in the variables $\mathbf{x}=\left(x_{1}, \ldots, x_{n}\right)$. A polynomial $p \in \mathbb{R}[\mathbf{x}]$ is a sum of squares (in short SOS) if $p$ can be written as:

$$
p(\mathbf{x})=\sum_{j \in J} p_{j}(\mathbf{x})^{2}, \quad \forall \mathbf{x} \in \mathbb{R}^{n},
$$

for some finite family of polynomials $\left\{p_{j}, j \in J\right\} \subset \mathbb{R}[\mathbf{x}]$. Notice that necessarily the degree of $p$ must be even, and also, the degree of each $p_{j}$ is bounded by half of that of $p$.

For a multi-index $\alpha \in \mathbb{N}^{n}$, let

$$
|\alpha|=\sum_{i=1}^{n} \alpha_{i}
$$

and define the vector:

$$
\mathbf{v}_{d}(\mathbf{x})=\left(\mathbf{x}^{\alpha}\right)_{|\alpha| \leq d}=\left(1, x_{1}, \ldots, x_{n}, x_{1}^{2}, x_{1} x_{2}, \ldots, x_{n-1} x_{n}, x_{n}^{2}, \ldots, x_{1}^{d}, \ldots, x_{n}^{d}\right),
$$

of all the monomials $\mathbf{x}^{\alpha}$ of degree less or equal to $d$ which has dimension $s(d)=\left(\begin{array}{c}n+d \\ d\end{array}\right)$. Those monomials form the canonical basis of the vector space $\mathbb{R}[\mathbf{x}]^{d}$ of $n$-variables polynomials of degree at most $d$. 
Proposition 2.5. A polynomial $p \in \mathbb{R}[\mathbf{x}]^{2 d}$ has a sum of square (SOS) decomposition if and only if there exists a real symmetric and positive semidefinite matrix $Q \in \mathbb{R}^{s(d) \times s(d)}$ such that

$$
p(\mathbf{x})=\mathbf{v}_{d}(\mathbf{x})^{\mathrm{T}} Q \mathbf{v}_{d}(\mathbf{x}), \forall \mathbf{x} \in \mathbb{R}^{n}
$$

Therefore, given a SOS polynomial $g \in \mathbb{R}[\mathbf{x}]^{2 d}$, the identity $g(\mathbf{x})=\mathbf{v}_{d}(\mathbf{x})^{\mathrm{T}} Q \mathbf{v}_{d}(\mathbf{x})$ provides linear equations that the coefficients of the matrix $Q$ must satisfy. Hence writing:

$$
\mathbf{v}_{d}(\mathbf{x})^{\mathrm{T}} \mathbf{v}_{d}(\mathbf{x})=\sum_{\alpha \in \mathbb{N}^{n}} B_{\alpha} \mathbf{x}^{\alpha}
$$

for appropriate $s(d) \times s(d)$ real symmetric matrix $B_{\alpha}$, checking whether the polynomial $g(\mathbf{x})=$ $\sum_{\alpha} g_{\alpha} \mathbf{x}^{\alpha}$ is SOS reduces to solving the SDP (feasibility) problem:

$$
\begin{gathered}
\text { Find } Q \in \mathbb{R}^{s(d) \times s(d)} \text {, such that: } \\
Q^{\mathrm{T}}=Q, \quad Q \succeq 0, \quad\left\langle Q, B_{\alpha}\right\rangle=g_{\alpha}, \quad \forall \alpha \in \mathbb{N}^{n} .
\end{gathered}
$$

a tractable convex optimization problem for which efficient software packages are available.

There are amazing ideas related to the above connection between positive polynomials and SDP. Using the so called moment problem nice hierarchies of tractable problems have been proposed to deal with, for instance, global optimization, see [86] and the references therein. Extending the idea to SOS-convexity $[5,4]$ new tractable relaxations have been proposed to problems in control. A last example is the new interest in classical Lyapunov's method for determining the stability of dynamical systems, specially by using SDP for finding polynomic Lyapunov's functions in polynomial differential equations.

In 1892 Lyapunov introduced his famous stability theory for nonlinear and linear systems. To be specific but no very technical, we recall that a dynamical system described by a homogeneous system of equations:

$$
\dot{x}=f(x), \quad \text { where } \dot{x}=\frac{d x}{d t}, \quad f: \mathbb{R}^{n} \rightarrow \mathbb{R}^{n}, \quad f\left(0_{n}\right)=0_{n},
$$

has a stable equilibrium point at $x=0_{n}$ if any solution $x\left(t, x_{0}\right)$ corresponding to an initial condition $x_{0}$ in some neightborhood of $0_{n}$, remains close to $0_{n}$ for all $t>0$. In the particular case when $x\left(t, x_{0}\right)$ converges to $0_{n}$ if $t \rightarrow+\infty$, the equilibrium is called asymptotically stable.

It is well known that stability can be certified if there exists a Lyapunov's function $V=V(x)$ such that,

$$
\begin{gathered}
V: \mathbb{R}^{n} \rightarrow \mathbb{R}, \\
V(x)>0, \forall x \neq 0_{n}, \quad V(0)=0, \\
\frac{d V(x(t))}{d t}=\nabla V(x(t)) \dot{x}(t)=\nabla V(x(t)) f(x(t)) \leq 0, \forall t>0 .
\end{gathered}
$$

and also asymptotical stability if furthermore the last inequality is strict. 
For a long time a computable general method to find Lyapunov's function were available only for the linear case:

$$
\dot{x}=A x,
$$

for example, in the form of a quadratic function:

$$
V(x)=x^{\mathrm{T}} P x
$$

satisfying

$$
\frac{d}{d t} V(x)=x^{\mathrm{T}}\left[A^{\mathrm{T}} P+P A\right] x=-x^{\mathrm{T}} Q x,
$$

where $P, Q$ are symmetric, positive definite $n \times n$ matrices. The matrix algebraic equation:

$$
A^{\mathrm{T}} P+P A=-Q
$$

is known as the Lyapunov algebraic equation. More about this important equation and its role in system stability and control can be found in [50].

There is no general procedure for finding the Lyapunov functions for nonlinear systems. In the last few decades however, advances in the theory and practice of convex optimization and in particular in semi-definite programming (SDP) have rejuvenated Lyapunov theory. The approach has been used to parameterize a class of Lyapunov functions with restricted complexity (e.g. quadratics, pointwise maximum of quadratics, polynomials, etc...) and then to pose the search of a Lyapunov function as a convex feasibility problem (see, for example [110], [109]).

Expanding on the concept of sum of squares decomposition of polynomials, this technique allows the formulation of semi-definite programs that search for polynomial Lyapunov functions for polynomial dynamical systems [3]. Sum of squares Lyapunov functions along with many others SDP based techniques, have also been applied to systems that undergo switching, e.g. ([124], [108], [119], [112]).

Perhaps so far it is not clear for the reader how the SDP problems arise in the context of dynamical systems stability and in the Lyapunov's function finding. But searching for a polynomic Lyapunov function for a polynomial dynamical system is reduced to find the coefficients of a $n$-variable polynomial $p\left(x_{1}, \ldots, x_{n}\right)$ of some degree $d$ such that the following polynomial inequalities hold:

$$
\begin{aligned}
p(x) & >0, \forall x \neq 0, \\
\frac{d}{d t} p[x(t)] & =\nabla p(x) f(x)<0 .
\end{aligned}
$$

In this case we have two polynomial inequalities, but the solution of the problem (7) which find a matrix $Q$ representing $p$ as a quadratic form of $\mathbf{v}_{d}(\mathbf{x})$ is not unique. In fact, it can be shown that the whole solution matrix set of the equations given by $p(\mathbf{x})=\mathbf{v}_{d}(\mathbf{x})^{\mathrm{T}} Q \mathbf{v}_{d}(\mathbf{x})$ is a linear space. When this linear space intersects the positive semi-definite matrix cone then $p(\mathbf{x})$ is SOS. 
In practice the general method searchs for a representation $Q$ of $p(\mathbf{x})=\mathbf{v}_{d}(\mathbf{x})^{\mathrm{T}} Q \mathbf{v}_{d}(\mathbf{x})$ and another quadratic representation $R$ of

$$
\frac{d}{d t} p[x(t)]=\nabla p(x) Q f(x)=\mathbf{v}_{d}(\mathbf{x})^{\mathrm{T}} R \mathbf{v}_{d}(\mathbf{x}) .
$$

Then, a sufficient condition for the dynamical system stability is that the following matrix inequality system holds:

$$
\left\{\begin{array}{l}
\mathbf{v}_{d}(\mathbf{x})^{\mathrm{T}} Q \mathbf{v}_{d}(\mathbf{x})>0 \\
\mathbf{v}_{d}(\mathbf{x})^{\mathrm{T}} R \mathbf{v}_{d}(\mathbf{x}) \leq 0
\end{array}, \forall \mathbf{x} \in \mathbb{R}^{n},\right.
$$

or equivalently, if the following $2 s(d) \times 2 s(d)$-matrix is positive (semi-)definite:

$$
\tilde{Q}=\left(\begin{array}{cc}
Q & 0 \\
0 & -R
\end{array}\right)
$$

In this case the feasibility problem (8) can be formulated in terms of the searching of matrix $\tilde{Q}$. In usual control theory language this is called a LMI (linear matrix inequality) problem (see [109]), and there exists efficient software to solve it, for instance SOSTOOL $[121,120]$.

Extensions of this kind of results to the so called switched and hybrid systems are developed in ([119]). A dynamical system is called switched/hybrid system if it can be written in the following form:

$$
\dot{x}=f_{i}(x), \quad i=1, \ldots, N,
$$

where $x$ is the continuous state, $i$ is a discrete state and $f_{i}(x)$ is the vector field describing the dynamics of the $i-t h$ mode/subsystem. Depending on how the discrete state $i$ evolves, the system (9) is categorized as a switched system, if for each $x \in \mathbb{R}^{n}$ only one $i$ is possible, or as a hybrid system, if for some $x \in \mathbb{R}^{n}$ multiple $i$ are possible.

In the case of switched system, the system is in $i-t h$ mode at time $t$ if

$$
x(t) \in X_{i}=\left\{x \in \mathbb{R}^{n}: g_{i k}(x) \geq 0, k=1, \ldots, m_{i}^{X}\right\} .
$$

Additionaly, the state space partition $\left\{X_{i}\right\}$ must satisfy $\bigcup_{i} X_{i}=\mathbb{R}^{n}$ and $\operatorname{int}\left(X_{i}\right) \cap \operatorname{int}\left(X_{j}\right)=\emptyset$, for $i \neq j$. A boundary $S_{i j}$ between $X_{i}$ and $X_{j}$ is defined analogously by

$$
S_{i j}=\left\{x \in \mathbb{R}^{n}: h_{i j 0}(x)=0, h_{i j k}(x) \geq 0, k=1, \ldots, m_{i j}^{S}\right\} .
$$

The stability analysis of switched polynomial system is based again in SOS decomposition, using piecewise polynomial Lyapunov functions. A typical result follows:

Theorem 2.6. Consider the switched system (9)-(11). Assume there exists polynomials $V_{i}(x)$, $c_{i j}(x)$ with $V(0)=0$ if $0 \in X_{i}$, and sum of squares $a_{i k}(x) \geq 0$ and $b_{i k}(x) \geq 0$, such that

$$
V_{i}(x)-\sum_{k=1}^{m_{i}^{X}} a_{i k}(x) g_{i k}(x)>0, \forall x \neq 0, \forall i=1, \ldots, N,
$$




$$
\begin{aligned}
\frac{\partial V_{i}}{\partial x} f_{i}(x)+\sum_{k=1}^{m_{i}^{X}} b_{i k}(x) g_{i k}(x) & <0, \forall x \neq 0, \forall i=1, \ldots, N, \\
V_{i}(x)+c_{i j}(x) h_{i j 0}(x)-V_{j}(x) & =0, \forall i \neq j
\end{aligned}
$$

then the origin of the state space is asymptotically stable. A Lyapunov function that prove this is the piecewise polynomial function $V(x)$, defined by:

$$
V(x)=V_{i}(x), \text { if } x \in X_{i} .
$$

The SOS polynomials $a_{i k}, b_{i k}$ at $X_{i}$ are computed using constrained feasibility SDP and LMI methods.

\subsection{Euclidean Distance Matrices}

Let us discuss some SDP relaxations of the Euclidean Distance Matrix Completion problem. A complete survey on the topic is provided in the recent Handbook [9]. We present some of the problems and results in $[82,83]$ and encourage the readers to look for details in the survey and the references therein.

A matrix $D \in \mathbb{S}^{n}$ is called an Euclidean Distance Matrix (EDM), if there exist vectors $p_{1}, \ldots$, $p_{n} \in \mathbb{R}^{r}$, such that

$$
D_{i j}=\left\|p_{i}-p_{j}\right\|_{2}^{2}, \quad \forall i, j=1, \ldots, n
$$

The smallest dimension $r$, where the above representation is possible is called the embedding dimension of $D$, denoted by $\operatorname{embdim}(D)$. Let us denote the set of all Euclidean Distance Matrices by $\mathcal{E}^{n}$.

From the vectors $p_{1}, \ldots, p_{n} \in \mathbb{R}^{r}$ we can define the so called Gramm matrix $Y \in \mathbb{S}^{n}$ as

$$
Y_{i j}=p_{i}^{T} p_{j}, \quad \forall i, j=1, \ldots, n
$$

It holds then the relation

$$
D_{i, j}=\left\|p_{i}-p_{j}\right\|_{2}^{2}=p_{i}^{T} p_{i}+p_{j}^{T} p_{j}-2 p_{i}^{T} p_{j}=Y_{i i}+Y_{j j}-2 Y_{i j}, \quad \forall i, j=1, \ldots, n
$$

Given a Matrix $Y \in \mathbb{S}^{n}$ the row vector formed with its diagonal is a mapping that shall be denoted by $\operatorname{diag}(Y)$. The adjoint operator of this mapping shall be called $\operatorname{Diag}(d)=\operatorname{diag}^{*}(d)$ and is obtained as the diagonal matrix with the vector $d$ along the diagonal. Further the row vector with all entries equal to one should be noted by $\mathbf{e}$. The last expression in the above relationship (12) can be intended as a mapping $\mathcal{K}: \mathbb{S}^{n} \rightarrow \mathbb{S}^{n}$, i.e.

$$
\mathcal{K}:=\operatorname{diag}(Y) \mathbf{e}^{T}+\mathbf{e} \operatorname{diag}(Y)^{T}-2 Y
$$

Using the linear map $\mathcal{K}$ the set of Euclidean Distance Matrices can be described as image of the cone of semidefinite constraints, i.e. $\mathcal{K}\left(\mathbb{S}^{n}\right)=\mathcal{E}^{n}$. There is an explicit representation of the Moore-Penrose generalized inverse of $\mathcal{K}$ as follows:

$$
\mathcal{K}^{\dagger}(D)=-\frac{1}{2}\left[I_{n}-\frac{1}{n} \mathbf{e e}^{T}\right][D-\operatorname{Diag}(\operatorname{diag}(D))]\left[I_{n}-\frac{1}{n} \mathbf{e e}^{T}\right] .
$$


The range spaces of $\mathcal{K}$ and $\mathcal{K}^{\dagger}$ are called the hollow space and the centered space (denoted as $\mathbb{S}_{H}^{n}$ and $\mathbb{S}_{C}^{n}$ ), respectively, and can be described as follows:

$$
\mathbb{S}_{H}^{n}=\left\{D \in \mathbb{S}^{n} \mid \operatorname{diag}(D)=0\right\}, \quad \mathbb{S}_{C}^{n}=\left\{Y \in \mathbb{S}^{n} \mid Y \mathbf{e}=0\right\} .
$$

The following relations are then useful

$$
\begin{gathered}
\mathcal{K}\left(\mathbb{S}_{C}^{n}\right)=\mathbb{S}_{H}^{n}, \quad \mathcal{K}^{\dagger}\left(\mathbb{S}_{H}^{n}\right)=\mathbb{S}_{C}^{n}, \quad \mathcal{K}\left(\mathbb{S}_{+}^{n} \cap \mathbb{S}_{C}^{n}\right)=\mathcal{E}^{n}, \quad \mathcal{K}^{\dagger}\left(\mathcal{E}^{n}\right)=\mathbb{S}_{+}^{n} \cap \mathbb{S}_{C}^{n} \\
D \in \mathcal{E}^{n} \Longleftrightarrow \mathcal{K}^{\dagger}(D) \in \mathbb{S}_{+}^{n}, \quad \operatorname{embdim}(D)=\operatorname{rank}\left(\mathcal{K}^{\dagger}(D)\right)
\end{gathered}
$$

If we restrict $\mathcal{K}$ and $\mathcal{K}^{\dagger}$ to the subspaces $\mathbb{S}_{C}^{n}$ and $\mathbb{S}_{H}^{n}$, respectively, then $\mathcal{K}$ is bijection and $\mathcal{K}^{\dagger}$ its inverse. Moreover, the restriction $\mathcal{K}: \mathbb{S}_{+}^{n} \cap \mathbb{S}_{C}^{n} \longrightarrow \mathcal{E}^{n}$ is also a bijection and $\mathcal{K}^{\dagger}$ : $\mathcal{E}^{n} \longrightarrow \mathbb{S}_{+}^{n} \cap \mathbb{S}_{C}^{n}$ its inverse. So far, the problem of deciding whether a given Matrix $D \in \mathbb{S}^{n}$ is an Euclidean distance matrix with embedding dimension not greater than $r$ can be stated as follows:

$$
\begin{aligned}
\text { Find } & Y \in \mathbb{S}_{+}^{n} \cap \mathbb{S}_{C}^{n} \\
\text { such that } & \mathcal{K}(Y)=D \\
& \operatorname{rank}(Y)=r
\end{aligned}
$$

or equivalently as

$$
\begin{aligned}
\min & 0 \\
\text { subject to } & \mathcal{K}(Y)=D \\
& Y \mathbf{e}=0 \\
& Y \succeq 0 \\
& \operatorname{rank}(Y)=r
\end{aligned}
$$

Deleting the last rank constraint we obtain an instance of the (SDP-P), where the Slater-CQ fails (due to the condition $Y \mathbf{e}=0$ ).

Consider now that for a matrix $D \in \mathbb{S}^{n}$ with zero diagonal and nonnegative elements some entries are known and other are not specified. Let us further assume that every specified principal submatrix of $D$ is an Euclidean distance matrix with embedding dimension less or equal to $r$. The Euclidean Distance Matrix Completion (EDMC) problem consists in finding the not specified entries of $D$, in such a way that $D$ is an Euclidean distance matrix. In order to specify the problem mathematically, let us associate to $D$ a 0 -1 matrix $H \in \mathbb{S}^{n}$ such that $H_{i j}=1$ for the specified entries of $D$ and $H_{i j}=0$ otherwise. Using the Hadamard component-wise product $\left((A \circ B)_{i j}=A_{i j} B_{i j}\right)$ the $(\mathrm{EDMC})$ problem for $D$ can be then written as

$$
\begin{aligned}
\text { Find } & \Gamma \in \mathcal{E}^{n} \\
\text { such that } & H \circ \Gamma=H \circ D
\end{aligned}
$$

The low dimensional Euclidean Distance Matrix Completion adds the constraint that the embedding dimension should not be smaller than $r$, i. e.

Find $\Gamma \in \mathcal{E}^{n}$

$$
\begin{array}{ll}
\text { such that } & H \circ \Gamma=H \circ D \\
& \operatorname{embdim}(\Gamma)=r
\end{array}
$$


This problem can be equivalently written as

$$
\begin{aligned}
\min & 0 \\
\text { subject to } & H \circ \mathcal{K}(Y)=H \circ D \\
& Y \mathbf{e}=0 \\
& Y \succeq 0 \\
& \operatorname{rank}(Y)=r
\end{aligned}
$$

and it is NP-hard. The relaxation obtained by deleting the rank constraint is a tractable SDP problem, but the solutions usually has too large values for $\operatorname{rank}(Y)$ and there are many different heuristics to improve this relaxation [83].

Another idea is to take advantage of the degeneracy (in the sense that the Slater-CQ fails) and to reduce the dimension of the problem using a proper semidefinite facial reduction. Given a subset $\alpha \subset\{1, \ldots, n\}$ and a matrix $Y \in \mathbb{S}^{n}$ let us denote by the principal submatrix of $Y$ formed from the rows and columns with index in $\alpha$ as $Y[\alpha]$. Based on this notation we can define for a fixed matrix $\bar{D} \in \mathcal{F}^{k}$, with $|\alpha|=k$ the set

$$
\mathcal{E}^{n}(\alpha, \bar{D})=\left\{D \in \mathcal{E}^{n} \mid D[\alpha]=\bar{D}\right\}
$$

For instance, if the fixed entries of the matrix $D$ in the above low dimensional (EDMC) problem are exactly those from the matrix formed by the first $k$ rows and columns, where the specified submatrix is $\bar{D} \in \mathcal{E}^{k}$ with $\operatorname{embdim}(\bar{D})=r$, then the low dimensional (EDMC) problem can be intended as to find one element in the set

$$
\left\{Y \in \mathbb{S}^{n} \mid Y \in \mathcal{K}^{\dagger}\left(\mathcal{E}^{n}(1: k, \bar{D})\right), \text { rank } Y=r\right\}
$$

Here we write MATLAB notation $1: k=\{1, \ldots, k\}$ for simplicity.

Theorem 2.7. Let $D \in \mathcal{E}^{n}$, with embedding dimension $r$. Let $\bar{D}=D[1: k] \in \mathcal{E}^{k}$ with embedding dimension $t$, and $B=\mathcal{K}^{\dagger}(\bar{D})=\bar{U}_{B} S \bar{U}_{B}$ for some $\bar{U}_{B} \in \mathbb{R}^{k \times t}$ with $\bar{U}_{B}^{T} \bar{U}_{B}=I_{t}$ and $S \in \mathbb{S}_{+}^{t}$ positive definite. Then

$$
\text { face } \mathcal{K}^{\dagger}\left(\mathcal{E}^{n}(1: k, \bar{D})\right)=\left(U \mathbb{S}_{+}^{n-k+t+1} U\right) \cap \mathbb{S}_{C}^{n}=(U V) \mathbb{S}_{+}^{n-k+t}(U V)^{T}
$$

where

$$
\begin{gathered}
U_{B}=\left[\bar{U}_{B} \frac{1}{\sqrt{n}} \boldsymbol{e}\right] \in \mathbb{R}^{k \times(t+1)} \\
U=\left[\begin{array}{cc}
U_{b} & 0 \\
0 & I_{n-k}
\end{array}\right] \in \mathbb{R}^{n \times(n-k+t+1)} \text { and }\left[V \frac{U^{T} \boldsymbol{e}}{\left\|U^{T} \boldsymbol{e}\right\|}\right]
\end{gathered}
$$

is an square orthogonal matrix of dimension $(n-k+t+1)$.

This remarkable result provides a reduction of the size of the (EDM) completion problem. Instead of working with matrices in $\mathbb{S}^{n}$, the problem is now stated with smaller matrices in $\mathbb{S}^{n-k+t}$. 
There is a natural way to associate a weighted undirected Graph $G=(N, E, \omega)$ to the (EDMC) problem defined by a matrix $D \in \mathbb{S}^{n}$ (with zero diagonal, nonnegative elements and specified and unspecified entries). In fact, taking the nodes set $N=\{1, \ldots, n\}$, the edge set $E=\{i j \mid i \neq$ $j, D_{i j}$ is specified $\}$ and the weights $\omega_{i j}=\sqrt{D_{i j}}$, for all $i j \in E$. In this setting the matrix $H$ used in (13) correspond just to the adjacency matrix of $G$. Moreover, a specified principal submatrix in $D$ can be interpreted as a clique in $G$. So far, the above result deals with the case of a single clique. It shows in particular, the equivalence to appropriated faces and opens the possibility to reduce the problem using information of a clique.

In [82] the above result is extended in many ways, first considering two (or more) disjoint cliques and then describing the faces associated to intersecting cliques. A deeper insight of the subsequent reduction of the problem can be taken from [82, 83], where this procedure is applied to the so called Sensor Network Localization Problem and numerical examples with the solution of large instances are discussed. The same technique of semidefinite facial reduction over cliques for EDMC problems have been successfully applied to other areas, see for instance $[15,12]$.

\section{NONLINEAR SEMIDEFINITE PROGRAMMING}

Let us consider in this section the following nonlinear semidefinite programming (NLSDP) model

$$
\begin{array}{lll}
(N L S D P) \quad \min & f(x) \\
& G(x) \preceq 0
\end{array}
$$

where the mappings $f: \mathbb{R}^{n} \longrightarrow \mathbb{R}$ and $G: \mathbb{R}^{n} \longrightarrow \mathbb{S}^{m}$ are in general smooth and nonlinear. Equality constraints can be also included in the (NLSDP) model, but for simplicity of presentation, we have chosen the above simple (NLSDP). In particular, all statements discussed in this section can be adapted to the case with equalities.

The (SDP) model of the previous section is already a nonlinear convex optimization problem. However in some important application problems, see e.g. [102, 100, 106], it is helpful to incorporate non convex and nonlinear functions into the model resulting in the above (NLSDP). More recently NLSDP has been used for modelling in new different applications areas like magnetic resonance tissue quantification [8], truss design and structural optimization [2, 17, 67, 73], material optimization [1, 74, 79, 129, 127, 128, 57, 78], passive reduced order modelling [48], fixed-order control design [10], finance $[80,88]$ and reduced order control design for PDE systems [91], among others.

The optimality conditions of first and second order for NLSDP are widely characterized, see for instance [21, 23, 35, 125, 47]. An important effort research is recently devoted to the study and characterization of stability for solutions of nonlinear semidefinite programming (or in general conic) problems, see for instance [107, 20, 33, 70, 49, 98, 97].

We present briefly the optimality condition for the model (NLSDP) and refer to [65] for a detailed discussion of the key differences to the usual case of nonlinear programming. 
The Lagrangian $\mathcal{L}: \mathbb{R}^{n} \times \mathbb{S}^{m} \rightarrow \mathbb{R}$ of (NLSDO) is defined by $\mathcal{L}(x, Y):=f(x)+\langle G(x), Y\rangle$. and its gradient with respect to $x$ can be written as

$$
\nabla_{x} \mathcal{L}(x, Y)=\nabla f(x)+D G(x)^{*}[Y]
$$

Here $D G(x)[]:. \mathbb{R}^{n} \longrightarrow \mathbb{S}^{m}$ is defined as

$$
D G(x)[d]=\sum_{i=1}^{n} d_{i} G_{i}(x), \text { with } G_{i}(x)=\frac{\partial G(x)}{\partial x_{i}}
$$

and the adjoint $D G(x)^{*}[]:. \mathbb{S}^{m} \longrightarrow \mathbb{R}^{n}$ is then

$$
D G(x)^{*}[Y]=\nabla_{x}\langle G(x), Y\rangle=\left(\left\langle G_{1}(x), Y\right\rangle, \ldots\left\langle G_{n}(x), Y\right\rangle\right)^{T} .
$$

The Mangasarian-Fromovitz constraint qualification is satisfied at the feasible point $x$ if there exists a vector $d \in \mathbb{R}^{n}$ such that $G(\bar{x})+D G(x)[d] \prec 0$.

Theorem 3.1. If $\bar{x}$ is a local minimizar of (NLSDP) where the Mangasarian-Fromovitz constraint qualification holds true, then there exist matrices $\bar{Y}, \bar{S} \in \mathbb{S}^{m}$ such that

$$
\begin{aligned}
G(\bar{x})+\bar{S} & =0, \\
\nabla f(\bar{x})+D G(\bar{x})^{*}[Y] & =0, \\
\bar{Y} \bar{S} & =0, \\
\bar{Y}, \bar{S} & \succeq 0 .
\end{aligned}
$$

A point $(\bar{x}, \bar{Y}, \bar{S})$ satisfying (15) is a stationary point of (NLSDP). For simplicity let us consider only the case that the above $\bar{Y}$ and $\bar{S}$ and unique and satisfy strict complementarity, i.e. $\bar{Y}+\bar{S} \succ 0$. In the following we state second order sufficient conditions due to [125]. Let us then consider a strict complementary stationary point $(\bar{x}, \bar{Y}, \bar{S})$. In this case the cone of critical directions at $\bar{x}$ can be written as follows, see e.g. [20,65],

$$
C(\bar{x}):=\left\{h \mid U_{1}^{T} D G(\bar{x})[h] U_{1}=0\right\},
$$

where $U=\left[U_{1}, U_{2}\right]$ is an unitary matrix that simultaneously diagonalizes $\bar{Y}$ and $\bar{S}$. Here also, $U_{2}$ has $r:=\operatorname{rank}(\bar{S})$ columns and $U_{1}$ has $m-r$ columns. Moreover the first $m-r$ diagonal entries of $U^{T} \bar{S} U$ are zero, and the last $r$ diagonal entries of $U^{T} \bar{Y} U$ are zero.

Let us denote the Hessian of the Lagrangian by

$$
\nabla_{x}^{2} \mathcal{L}(x, Y)=\nabla^{2} f(x)+D^{2} G(x)^{*}[Y]
$$

where $D^{2} G(x)^{*}[Y]=\nabla_{x}^{2}\langle G(x), Y\rangle$

The second order suffcient condition is satisfied at $\bar{x}, \bar{Y}$ if

$$
h^{T}\left(\nabla_{x}^{2} \mathcal{L}(\bar{x}, \bar{Y})+\mathcal{H}(\bar{x}, \bar{Y})\right) h>0 \quad \forall h \in C(\bar{x}) \backslash\{0\}
$$


Here $\mathcal{H}$ is a nonnegative matrix related to the curvature of the semidefinite cone in $G(\bar{x})$ along direction $\bar{Y}$ (see [125]) and is given by its matrix entries

$$
\mathcal{H}_{i, j}:=-2\left\langle\bar{Y}, G_{i}(\bar{x}) G(\bar{x})^{\dagger} G_{j}(\bar{x})\right\rangle,
$$

where $G(\bar{x})^{\dagger}$ denotes the Moore-Penrose pseudo-inverse of $G(\bar{x})$.

Theorem 3.2. Let $(\bar{x}, \bar{Y}, \bar{S})$ be a stationary point of (NLSDP) satisfying strict complementarity. If the second order sufficient condition holds true, then $\bar{x}$ is a strict local minimizer.

The following very simple example of [43] shows that the classical second order sufficient condition, i.e.

$$
h^{T}\left(\nabla_{x}^{2} \mathcal{L}(\bar{x}, \bar{Y})\right) h>0 \quad \forall h \in C(\bar{x}) \backslash\{0\}
$$

is generally too strong in the case of semidefinite constraints, since it does not exploit curvature of the non-polyhedral semidefinite cone.

$$
\begin{array}{ll}
\min _{x \in \mathbb{R}^{2}} & -x_{1}-\left(x_{2}-1\right)^{2} \\
\text { s.t. } & {\left[\begin{array}{rrr}
-1 & 0 & -x_{1} \\
0 & -1 & -x_{2} \\
-x_{1} & -x_{2} & -1
\end{array}\right] \preceq 0}
\end{array}
$$

It is a trivial task to check that the constraint $G(x) \preceq 0$ is equivalent to the inequality $x_{1}^{2}+$ $x_{2}^{2} \leq 1$, such that $\bar{x}=(0,-1)^{T}$ is the global minimizer of the problem.

The first order optimality conditions (15) are satisfied at $\bar{x}$ with associated multiplier

$$
\bar{Y}=\left[\begin{array}{lll}
0 & 0 & 0 \\
0 & 2 & 2 \\
0 & 2 & 2
\end{array}\right] .
$$

Strict complementarity condition also holds true. The Hessian of the Lagrangian at $(\bar{x}, \bar{Y})$ for this problem can be calculated as

$$
\nabla_{x x}^{2} \mathcal{L}(\bar{x}, \bar{Y})=\left[\begin{array}{rr}
-2 & 0 \\
0 & -2
\end{array}\right] .
$$

It is negative definite, and the stronger second order condition is not satisfied.

The orthogonal matrix

$$
U=\left[\begin{array}{ccc}
1 & 0 & 0 \\
0 & \frac{1}{\sqrt{2}} & \frac{1}{\sqrt{2}} \\
0 & -\frac{1}{\sqrt{2}} & \frac{1}{\sqrt{2}}
\end{array}\right]
$$


simultaneously diagonalizes $\bar{Y}, G(\bar{x})$ and the Moore-Penrose pseudoinverse matrix at $\bar{x}$ is then given by

$$
G(\bar{x})^{\dagger}=\frac{-1}{4}\left[\begin{array}{rrr}
4 & 0 & 0 \\
0 & 1 & -1 \\
0 & -1 & 1
\end{array}\right]
$$

Consequently the matrix associated to the curvature becomes

$$
\mathcal{H}(\bar{x}, \bar{Y})=\left[\begin{array}{ll}
4 & 0 \\
0 & 0
\end{array}\right] .
$$

Finally, the cone of critical directions has the form $h=\left(h_{1}, 0\right)^{T}$ with $h_{1} \in \mathbb{R}$ and then the weaker second order sufficient condition holds true.

The most developed general algorithmic approach for NLSDP is the one due to Kočvara and Stingl, see [71, 72, 73, 75, 76, 126]. It bases on generalized augmented Lagrangians designed for the semidefinite constraint and solves a sequence of unconstrained minimization problems driven by a penalty parameter. There are other approaches for dealing with general NLSDP, for instance, sequential semidefinite programming [46, 48, 36, 56, 51, 52, 131, 139, 43], bundle methods [104, 105], partially augmented Lagrangian approach [10, 45, 106], interior point trust region [89, 90, 91], predictor-corrector interior point [64], augmented Lagrangian [106, 132], successive linearization [68] and primal-dual interior point methods [142, 143, 69] among others. There is not a definitive answer to the question of which is the most convenient approach for solving NLSDP in general, which explains the intense research activity going on in this area.

\section{CONCLUDING REMARKS}

The various recent developments in SDP connecting to new areas of mathematics are in our opinion a strong evidence, that this topic remains a promising research area. It will be for sure in the next years a beautiful source of new interesting applications as well as theoretical results.

\section{ACKNOWLEDGMENTS}

We thank the referee for the helpful suggestions and comments. This work was partially supported by Conicyt Chile under the grant Fondecyt Nr. 1120028.

\section{REFERENCES}

[1] AchtZiger W \& KočVARA M. 2007. On the maximization of the fundamental eigenvalue in topology optimization. Structural and Multidisciplinary Optimization, 34(3): 181-195.

[2] ACHTZiger W \& KoČVARA M. 2007. Structural topology optimization with eigenvalues. SIAM Journal on Optimization, 18(4): 1129-1164.

[3] Ahmadi AA. 2011. Algebraic relaxations \& hardness results in polynomial optimization \& lyapunov analysis. Ph.D. Massachusetts Institute of Technology. 
[4] Ahmadi AA \& PARrilo PA. 2012. A convex polynomial that is not sos-convex. Mathematical Programming, 135(1-2): 275-292.

[5] AhMAdi AA \& PARRILO PA. 2013. A complete characterization of the gap between convexity and sosconvexity. SIAM Journal on Optimization, 23(2): 811-833.

[6] Alizadeh F. 1995. Interior point methods in semidefinite programming with applications to combinatorial optimization. SIAM Journal on Optimization, 5(1): 13-51.

[7] Alizadeh F, Haeberly JA \& Overton M. 1997. Complementarity and nondegeneracy in semidefinite programming. Mathematical Programming, 77(1): 111-128.

[8] Anand Ch K, Sotirov R, Terlaky T \& Zheng Z. 2007. Magnetic resonance tissue quantification using optimal bssfp pulse-sequence design. Optimization and Engineering, 8(2): 215-238.

[9] Anjos MF \& Lasserre JB (EDS.). 2012. Handbook on Semidefinite, Conic and Polynomial Optimization. International Series in Operations Research \& Management Science, Vol. 166, Springer.

[10] Apkarian P, Noll D \& TUAN D. 2003. Fixed-order H1 control design via a partially augmented lagrangian method. Int. J. Robust Nonlinear Control, 13(12): 1137-1148.

[11] ARTIN E. 1927. Über die zerlegung definiter funktionen in quadrate. Abhandlungen aus dem Mathematischen Seminar der Universitt Hamburg, 5(1): 100-115.

[12] BABAK A, Krislock N, GHodsi A \& Wolkowicz H. 2012. Large-scale manifold learning by semidefinite facial reduction. Technical Report, Universiy of Waterloo.

[13] Babak A, Krislock N, Ghodsi A, Wolkowicz H, Donaldson L \& Li M. 2011. Spros: An sdp-based protein structure determination from $\mathrm{nmr}$ data. Technical Report, Universiy of Waterloo.

[14] Babak A, Krislock N, Ghodsi A, Wolkowicz H, Donaldson L \& Li M. 2012. Protein structure by semidefinite facial reduction. In Benny Chor, editor, Research in Computational Molecular Biology, volume 7262 of Lecture Notes in Computer Science, Springer, pages 1-11.

[15] Babak A, Krislock N, Ghodsi A, Wolkowicz H, Donaldson L \& Li M. 2013. Determining protein structures from NOESY distance constraints by semidefinite programming. Journal of Computational Biology, 40(4): 296-310.

[16] Ben-Tal A, El Ghaoui L \& Nemirovski AS. 2009. Robust Optimization. Princeton Series in Applied Mathematics. Princeton University Press, October.

[17] Ben-Tal A, Jarre F, KoČVara M, Nemirovs ki A \& Zowe J. 2000. Optimal design of trusses under a nonconvex global buckling constraint. Optimization and Engineering, 1(2): 189-213.

[18] Ben-Tal A \& Nemirovski A. 2001. Lectures on Modern Convex Optimization. MPS-SIAM, Series on Optimization. SIAM Philadelphia.

[19] Blekherman G, Parrilo PA \& Thomas R. 2012. Semidefinite Optimization and ConvexAlgebraic Geometry. MPS-SIAM, Series on Optimization Vol. 13. SIAM.

[20] Bonnans F \& H RAmíRez C. 2005. Strong regularity of semidefinite programming problems. Informe Técnico, DIM-CMM, Universidad de Chile, Santiago, Departamento de Ingeniería Matemática, No CMM-B-05/06-137.

[21] Bonnans JF \& Shapiro A. 2000. Perturbation analysis of optimization problems. SpringerVerlag, New York.

[22] BonnAns JF \& SHAPIRO A. 2003. Nondegeneracy and quantitative stability of parameterized optimization problems with multiple solutions. SIAM Journal on Optimization, 8(4): 940-946. 
[23] Bonnans JF, Cominetti R \& Shapiro A. 1999. Second order optimality conditions based on parabolic second order tangent sets. SIAM Journal on Optimization, 9(2): 466-492.

[24] BORWEIN JM \& WOLKOWICZ H. 1980/81. Characterization of optimality for the abstract convex program with finite dimensional range. J. Australian Mathematical Society, Ser. A, 30(4): 390-411.

[25] BORWEIN JM \& WOLKOWICZ H. 1980/81. Facial reduction for a convex-cone programming problem. J. Australian Mathematical Society, Ser. A, 30(3): 369-380.

[26] Borwein JM \& Wolkowicz H. 1981. Regularizing the abstract convex program. J. Math. Anal. Appl., 83(2): 495-530.

[27] BosE NK \& LI CC. 1968. A quadratic form representation of polynomials of several variables and its applications. IEEE Transactions on Automatic Control, 13(4): 447-448.

[28] Boyd S, Ghaoui LE, Feron E \& Balakrishnan V. 1994. Linear Matrix Inequalities in system and control theory. SIAM Studies in Applied Mathematics. Vol. 15., SIAM, Philadelphia.

[29] Boyd S \& Vandenberghe L. 2004. Convex optimization. Cambridge University Press.

[30] BURER S \& MONTEIRO RDC. 2003. A nonlinear programming algorithm for solving semidefinite programs via low-rank factorization. Mathematical Programming, 95(2): 329-357.

[31] Burer S, Monteiro RDC \& Zhang Y. 2002. Solving a class of semidefinite programs via nonlinear programming. Mathematical Programming, 93(1): 97-122.

[32] Burkowski F, Cheung YL \& Wolkowicz H. 2013. Efficient use semidefinite programming for selection of rotamers in protein conformations. University of Waterloo, Department of Combinatorics and Optimization.

[33] CHAN ZX \& SUN D. 2008. Constraint nondegeneracy, strong regularity and nonsingularity in semidefinite programming. SIAM Journal on Optimization, 19: 370-396.

[34] Cheung Y-L, Schurr S \& Wolkowicz H. 2011. Preprocesing and regularization for degenerate semidefinite programs. Research Report CORR 2011-02, University of Waterloo.

[35] CominetTi R. 1990. Metric regularity, tangent sets and second order optimality conditions. Appl. Math. Optim., 21(3): 265-287.

[36] Correa R \& H RAMírez C. 2004. A global algorithm for nonlinear semidefinite programming. SIAM Journal on Optimization, 15(1): 303-318.

[37] DE KLERK E. 2002. Aspects of semidefinite programming. Interior point algorithms and selected applications. Applied Optimization. 65. Dordrecht, Kluwer Academic Publishers.

[38] DE KLERK E. 2010. Exploiting special structure in semidefinite programming: A survey of theory and applications. European Journal of Operational Research, 201(1): 1-10.

[39] De Klerk E, De Oliveira Filho FM \& Pasechnik DV. 2012. Relaxations of combinatorial problems via association schemes. In: M.F. Anjos \& J.B. Lasserre (eds.), Handbook of semidefinite, Conic and Polynomial Optimization, International Series in Operations Research and Management Science, 166: 171-199.

[40] De Klerk E, PASechnik DV \& Sotirov R. 2008. On semidefinite programming relaxations of the trvelling salesman problem. SIAM Journal on Optimization, 19(4): 1559-1573.

[41] DE KLERK E, Roos C \& TERLAKy T. 1997. Initialization in semidefinite programming via selfdual skewsymmetric embedding. Operations Research Letters, 20: 213-221.

[42] Delzell CN. 1984. A continuous constructive solution to Hilbert's 17th problem. Inventiones mathematicae, 76(3): 365-384. 
[43] Diehl M \& F JARRE CH Vogelbusch. 2006. Loss of superlinear convergence for an sqp-type method with conic constraints. SIAM Journal on Optimization, 16(4): 1201-1210.

[44] DÜR M, Jargalsaikhan B \& Still G. 2012. The slater condition is generic in linear conic programming. Optimization Online, November.

[45] Fares B, ApKarian P \& Noll D. 2001. An augmented lagrangian method for a class of lmiconstrained problems in robust control theory. Int. J. Control, 74(4): 348-360.

[46] FARES B, NOLL D \& APKARIAN P. 2002. Robust control via sequential semidefinite programming. SIAM Journal on Control and Optimization, 40(6): 1791-1820.

[47] Forsgren A. 2000. Optimality conditions for nonconvex semidefinite programming. Mathematical Programming, 88(1, Ser. A): 105-128.

[48] FReund RW \& F JARRE CH Vogelbusch. 2007. Nonlinear semidefinite programming: sensitivity, convergence, and an application in passive reduced-order modeling. Mathematical Programming, 109(2-3 B): 581-611.

[49] FUSEK P. 2012. On metric regularity for weakly almost piecewise smooth functions and some applications in nonlinear semidefinite programming. Optimization Online, December.

[50] Gajic Z \& Qureshi M (editors). 1995. Lyapunov Matrix Equation in System Stability and Control. Mathematics in Science and Engineering Series Vol 195, Academic Press, San Diego, California.

[51] GARCÉS R, GÓMEZ W \& JARRE F. 2011. A self-concordance property for nonconvex semidefinite programming. Mathematical Methods of Operations Research, pages 1-16.

[52] GARCÉS R, GómEZ W \& JARRE F. 2012. A sensitivity result for quadratic semidefinite programs with an application to a sequential quadratic semidefinite programming algorithm. Computational and Applied Mathematics, 31(1): 205-218.

[53] Goemans MX \& Williams On DP. 1995. Improved approximation algorithms for maximum cut and satisfiability problems using semidefinite programming. J. Assoc. Comput. Mach., 42(6): 11151145 .

[54] Goldman AJ \& TUCKeR AW. 1956. Theory of linear programming. In: H.W. Kuhn \& A.W. Tucker (eds.), Linear Inequalities and Related Systems, Annals of Mathematical Studie, Princeton University Press, Princeton NJ, 38: 63-97.

[55] Gómez W \& Gómez JA. 2006. Cutting plane algorithms for robust conic convex optimization problems. Optimization Methods and Software, 21(5): 779-803.

[56] Gómez W \& RAMíRez H. 2010. A filter algorithm for nonlinear semidefinite programming. Computational and Applied Mathematics, 29(2): 297-328.

[57] Haslinger J, KoČVara M, Leugering G \& Stingl M. 2010. Multidisciplinary free material optimization. SIAM Journal on Applied Mathematics, 70(7): 2709-2728.

[58] Helmberg C. 2002. Semidefinite programming. European Journal of Operational Research, 137(3): 461-482.

[59] Helmberg C \& Rendl F. 2000. A spectral bundle method for semidefinite programming. SIAM Journal on Optimization, 10(3): 673-696.

[60] Helmberg C, Rendl F, Vanderbei RJ \& Wolkowicz H. 1996. An interior-point method for semidefinite programming. SIAM Journal on Optimization, 6(2): 342-361. 
[61] Henrion D \& LASSERRE J-B. 2005. Detecting global optimality and extracting solutions in gloptipoly. In: D. Henrion \& A. Garulli (eds.), Positive Polynomials in Control, Lecture Notes in Control and Information Science Vol 312, Springer, pages 293-310.

[62] Henrion D, LAsserre JB \& LöfBerg J. 2009. Gloptipoly 3: moments, optimization and semidefinite programming. Optimization Methods and Software, 24(4-5): 761-779.

[63] JARRE F. 1993. An interior-point method for minimizing the maximum eigenvalue of linear combination of matrices. SIAM Journal on Control and Optimization, 31(5): 1360-1377.

[64] F. JARRE. 2000. An interior method for nonconvex semidefinite programs. Optimization and Engineering, 1(4): 347-372.

[65] JARRE F. 2012. Elementary optimality conditions for nonlinear sdps. In: M.F. Anjos \& J.B. Lasserre (eds.), Handbook of semidefinite, Conic and Polynomial Optimization, International Series in Operations Research and Management Science, Springer, 166: 455-470.

[66] JARRE F \& RENDL F. 2008. An augmented primal-dual method for linear conic programs. SIAM Journal on Optimization, 19(2): 808-823.

[67] Kanno Y \& TAKewaKi I. 2006. Sequential semidefinite program for maximum robustness design of structures under load uncertainty. Journal of Optimization Theory and Applications, 130(2): 265-287.

[68] Kanzow C, Nagel C, Kato H \& Fukushima M. 2005. Successive linearization methods for nonlinear semidefinite programs. Computational Optimization and Applications, 31: 251-273.

[69] Kato A, YABE H \& Yamashita H. 2013. An interior point method with a primal-dual quadratic barrier penalty function for nonlinear semidefinite programming. Optimization Online, February.

[70] Klatte D \& Kummer B. 2012. Aubin property and uniqueness of solutions in cone constrained optimization. Preprint, Professur Mathematik für Ökonomen, IBW, University of Zurich.

[71] KoČVARA M \& STINGL M. 2003. Pennon: a code for convex nonlinear and semidefinite programming. Optimization Methods and Software, 18(3): 317-333.

[72] KoČVARA M \& Stingl M. 2003. Pennon: a generalized augmented lagrangian method for semidefinite programming. In: Di Pillo, Gianni (ed.) et al., High performance algorithms and software for nonlinear optimization, Boston, MA: Kluwer Academic Publishers. Appl. Optim., 82: 303-321.

[73] KoČVARA M \& Stingl M. 2004. Solving nonconvex sdp problems of structural optimization with stability control. Optimization Methods and Software, 19(5): 595-609.

[74] KoČVARa M \& Stingl M. 2007. Free material optimization for stress constraints. Structural and Multidisciplinary Optimization, 33(4-5): 323-335.

[75] KočVARa M \& Stingl M. 2007. On the solution of large-scale sdp problems by the modified barrier method using iterative solvers. Mathematical Programming, 109(2-3 (B)): 413-444.

[76] KočVara M \& Stingl M. 2009. On the solution of large-scale sdp problems by the modified barrier method using iterative solvers: Erratum. Mathematical Programming, 120(1): 285-287.

[77] KočVARA M \& STIngl M. 2012. Pennon: Software for linear and nonlinear matrix inequalities. In: M.F. Anjos \& J.B. Lasserre (eds.), Handbook of semidefinite, Conic and Polynomial Optimization, International Series in Operations Research and Management Science, Springer, 166: 755791. 
[78] KoČVARA M \& Stingl M. 2012. Solving stress constrained problems in topology and material optimization. Structural and Multidisciplinary Optimization, 46(1): 1-15.

[79] Kočvara M, Stingl M \& Zowe J. 2008. Free material optimization: Recent progress. Optimization, 57(1): 79-100..

[80] KonNo H, KaWADAi N \& WU D. 2004. Estimation of failure probability using semidefinite logit model. Computational Management Science, 1: 59-73.

[81] Krishnan K \& Mitchell JE. 2003. Semi-infinite linear programming approaches to semidefinite programming problems. In: P. Pardalos \& H. Wolkowicz (eds.), Novel approaches to hard discrete optimization problems Fields Institute Communications Series, 37: 123-142.

[82] KRISLOCK N \& Wolkowicz H. 2010. Explicit sensor network localization using semidefinite representations and facial reductions. SIAM Journal on Optimization, 20(5): 2679-2708.

[83] Krislock N \& Wolkowicz H. 2012. Euclidean distance matrices and applications. In: M.F. Anjos \& J.B. Lasserre (eds.), Handbook of semidefinite, Conic and Polynomial Optimization, International Series in Operations Research and Management Science, Springer, 166: 879-914.

[84] LASSERRE JB. 2001. Global optimization with polynomials and the problem of moments. SIAM Journal on Optimization, 11(3): 756-817.

[85] LASSERRE JB. 2008. A semidefinite programming approach to the generalized problem of moments. Mathematical Programming, 112(1): 65-92.

[86] Lasserre JB. 2010. Moments, Polynomials and their Applications. Imperial College Press Optimization Series, Vol. 1, Imperial College Press, London.

[87] Laurent M \& Rendl F. 2005. Semidefinite programming and integer programming. In: $K$. Aardal, G. Nemhauser \& R. Weismantel (eds.), Handobook of Discrete Optimization, Elsevier, Amsterdam, pages 393-514.

[88] Leibfritz F \& MARUhn JH. 2009. A successive sdp-nsdp approach to a robust optimization problem in finance. Journal of Computational Optimization and Applications, 44(3): 443-466.

[89] Leibfritz F \& Mostafa ME. 2002. An interior point constrained trust region method for a special class of nonlinear semidefinite programming problems. SIAM Journal on Optimization, 12(4): 1048-1074.

[90] LeibFritz F \& Mostafa MF. 2003. Trust region methods for solving the optimal output feedback design problem. Int. J. Control, 76(5): 501-519.

[91] LEIBFRITZ F \& VOLKWEIN S. 2006. Reduced order output feedback control design for pde systems using proper orthogonal decomposition and nonlinear semidefinite programming. Linear Algebra and its Applications, 415(2-3): 542-575.

[92] LEwIS A. 2003. The mathematics of eigenvalue optimization. Mathematical Programming, 97(12): $155-176$.

[93] LÖFBERG J. 2004. YALMIP: A toolbox for modeling and optimization in MATLAB. In CCA/ ISIC/CACSD, September.

[94] Mittelmann H. 2012. The state-of-the-art in conic optimization software. In: M.F. Anjos \& J.B. Lasserre (eds.), Handbook of semidefinite, Conic and Polynomial Optimization, International Series in Operations Research and Management Science, Springer, 166: 671-686.

[95] Monteiro RDC. 2003. First- and second-order methods for semidefinite programming. Mathematical Programming, 97(1-2): 209-244. 
[96] MonteIRo RDC \& ZHANG Y. 1998. A unified analysis for a class of path-following primal-dual interiorpoint algorithms for semidefinite programming. Mathematical Programming, 81(3): 281299.

[97] Mordukhovich B, Tran N \& Ramírez H. 2013. Second-order variational analysis in conic programming with applications to optimality and stability. Optimization Online, January.

[98] Mordukhovich B, Tran N \& Rockafellar T. 2013. Full stability in finite-dimensional optimization. Optimization Online, July.

[99] Mosheyev L \& Zibulevsky M. 2000. Penalty/barrier multiplier algorithm for semidefinite programming. Optimization Methods and Software, 13(4): 235-261.

[100] Nemirovs KII A. 1993. Several np-hard problems arising in robust stability analysis. Math. Control, Signals Syst., 6(2): 99-105.

[101] NeSTEROV Y \& NeMIROVSKII A. 1994. Interior-point polynomial algorithms in convex programming. SIAM Stud. Appl. Math., 13, SIAM Philadelphia.

[102] NeSTEROV YE. 1998. Semidefinite relaxation and nonconvex quadratic optimization. Optimization Methods and Software, 9(1-3): 141-160.

[103] NeSTEROV YE \& TODD MJ. 1998. Primal-dual interior-point methods for self-scaled cones. SIAM Journal on Optimization, 8(2): 324-364.

[104] Noll D \& ApKaRian P. 2005. Spectral bundle methods for non-convex maximum eigenvalue functions: first-order methods. Mathematical Programming, 104(2-3, Ser. B): 701-727.

[105] Noll D \& ApKarian P. 2005. Spectral bundle methods for non-convex maximum eigenvalue functions: second-order methods. Mathematical Programming, 104(2-3, Ser. B): 729-747.

[106] Noll D, TORKi M \& APKARIAN P. 2004. Partially augmented lagrangian method for matrix inequality constraints. SIAM Journal on Optimization, 15(1): 181-206.

[107] PANG J-S, SUn D \& SUn J. 2003. Semismooth homeomorphisms and strong stability of semidefinite and lorentz complementarity problems. Mathematics in Operations Research, 28: 39-63.

[108] Papachristodoulou A \& Prajna S. 2002. On the construction of lyapunov functions using the sum of squares decomposition. Proceedings of the 41st IEEE Conference on Decision \& Control, pages 3482-3487.

[109] PARRILO PA. 2000. On a decomposition of multivariable forms via lmi methods. American Control Conference, 1(6): 322-326.

[110] PARRILO PA. 2000. Structured semi-definite programs and semialgebraic geometry methods in robustness and optimization. Ph.D. California Institute of Technology.

[111] PARRILO PA. 2003. Semidefinite relaxations for semialgebraic problems. Mathematical Programming, 96(2): 293-320.

[112] PARRILO PA \& JADBABAIE A. 2008. Approximation of a joint spectral radius using sum of squares. Linear Algebra Appl., 428(10): 2385-2402.

[113] PATAKi G. 1998. On the rank of extreme matrices in semidefinite programs and the multiplicity of optimal eigenvalues. Mathematics of Operations Research, 23(2): 339-358.

[114] Pataki G. 2000. The geometry of semidefinite programming. In: H. Wolkowicz, R. Saigal \& L. Vandenberghe (eds.), Handbook of Semidefinite Programming: Theory, Algorithms and Applications, Kluwer Academic Publishers. 
[115] PATAKi G. 2013. Strong duality in conic linear programming: facial reduction and extended duals. Department of Statistics and Operations Research, University of North Carolina at Chapel Hill. http://arxiv.org/abs/1301.7717v3.

[116] Peng J, Roos C \& Terlaky T. 2002. Self-regularity: A new paradigm for primal-dual interior points algorithms. Princeton Series in Apllied Mathematics, Princeton University Press.

[117] Povh J, Rendl F \& Wiegele A. 2006. A boundary point method to solve semidefinite programs. Computing, 78(3): 277-286.

[118] Powers V. 2011. Positive polynomials and sums of squares: Theory and practice. Department of Mathematics and Computer Science, Emory University, Atlanta.

[119] Prajna S \& Papachristodoulou A. 2003. Analysis of switched and hybrid systems - beyond piecewise quadratic methods. In Proceedings of the American Control Conference, pages 27792784.

[120] Prajna S, Papachristodoulou A \& Seiler P. 2005. Sostools and its control applications. In: D. Henrion and A. Garulli (eds.), Positive Polynomials in Control, Lecture Notes in Control and Information Science Vol 312, Springer, pages 273-292.

[121] Prajna S, Papachristodoulou A, Seiler P \& Parrilo PA. 2002-2005. SOSTOOLS: Sum of squares optimization toolbox for MATLAB.

[122] Ramana MV. 1997. An exact duality theory for semidefinite programming and its complexity implications. Mathematical Programming, 77(2): 129-162.

[123] Ramana MV, Tunçel L \& Wolkowicz H. 1997. Strong duality for semidefinite programming. SIAM Journal on Optimization, 7(3): 641-662.

[124] RANTZER MJA. 1998. Computation of piecewise quadratic lyapunov functions for hybrid systems. IEEE Trans. Automat. Control, 43(4): 555-559.

[125] Shapiro A. 1997. First and second order analysis of nonlinear semidefinite programs. Mathematical Programming, 77(2, Ser. B): 301-320.

[126] Stingl M. 2006. On the Solution of Nonlinear Semidefinite Programs by Augmented Lagrangian Methods. Dissertation, Shaker Verlag, Aachen.

[127] Stingl M, Kočvara M \& Leugering G. 2009. Free material optimization with fundamental eigenfrequency constraints. SIAM Journal on Optimization, 20(1): 525-547.

[128] Stingl M, KočVara M \& Leugering G. 2009. A new non-linear semidefinite programming algorithm with an application to multidisciplinary free material optimization. In: K. Kunisch, G. Leugering, J. Sprekels \& F. Tröltzsch (eds.), Optimal Control af coupled systems of partial differential equations, International Series of Numerical Mathematics 133: 275-295.

[129] Stingl M, KočVAra M \& LeUgering G. 2009. A sequential convex semidefinite programming algorithm with an application to multiple-load free material optimization. SIAM Journal on Optimization, 20(1): 130-155.

[130] STURM JF. 1999. Using SeDuMi 1.02, a MATLAB toolbox for optimization over symmetric cones. Optimization Methods and Software, 11/12(1-4): 625-653. See http://sedumi.ie.lehigh.edu.

[131] SUN D. 2006. The strong second-order sufficient condition and constraint nondegeneracy in nonlinear semidefinite programming and their implications. Math. Oper. Res., 31(4): 761-776.

[132] SUN D, SUN J \& ZHANG L. 2008. The rate of convergence of the augmented lagrangian method for nonlinear semidefinite programming. Mathematical Programming, 114(2, Ser. A): 349-391. 
[133] Todd MJ. 2001. Semidefinite optimization. Acta Numer., 10: 515-560.

[134] Toн KC, Todd MJ \& TÜTÜNCÜ RH. 2012. On the implementation and usage of sdpt3- a Matlab software package for semidefinite-quadratic-linear programming, version 4.0. In: M.F. Anjos \& J.B. Lasserre (eds.), Handbook of semidefinite, Conic and Polynomial Optimization, International Series in Operations Research and Management Science, Springer, 166: 715-753.

[135] TunçEl L \& Wolkowicz H. 2012. Strong duality and minimal representations for cone optimization. Computational Optimization and Applications, 53(2): 619-648.

[136] TÜTÜNCÜ RH, TOH KC \& TODD MJ. 2003. Solving semidefinite-quadratic-linear programs using sdpt3. Mathematical Programming, 95(2 Ser.B): 189-217.

[137] VAndenberghe L \& Boyd S. 1996. Semidefinite programming. SIAM Review, 38(1): 49-95.

[138] Vandenberghe L \& Boyd S. 1999. Applications of semidefinite programming. Applied Numerical Mathematics, 29(3): 283-299. Proceedings of the StieltjesWorkshop on High Performance Optimization Techniques.

[139] WANG Y, ZHANG SH \& ZHANG L. 2008. A note on convergence analysis of an sqp-type method for nonlinear semidefinite programming. Journal of Inequalities and Applications, vol. 2008, Article ID 218345, 10 pages, doi: 10.1155/2008/218345,2008: Article ID 218345, doi: 10.1155/2008/218345.

[140] Wolkowicz H \& ANJos MF. 2002. Semidefinite programming for discrete optimization and matrix completion problems. Discrete Appl. Math., 123(1-2): 513-577.

[141] Wolkowicz H, Saigal R \& Vandenberghe L (editors). 2000. Handbook of Semidefinite Programming: Theory, Algorithms and Applications. Kluwer's International Series in Operations Research and Management Science.

[142] YAMAShitA H \& YABE H. 2012. Local and superlineal convergence of a primal-dual interior point method for nonlinear semidefinite programming. Mathematical Programming, 132(1): 1-30.

[143] Yamashita H, Yabe H \& Harada K. 2012. A primal-dual interior point method for nonlinear semidefinite programming. Mathematical Programming, 135(1): 89-121.

[144] Yamashita M, Fujisawa K, Fukuda M, Kobayashi K, Nakata K \& Nakata M. 2012. Latest developments in th sdpa family for solving large-scale sdps. In: M.F. Anjos \& J.B. Lasserre (eds.), Handbook of semidefinite, Conic and Polynomial Optimization, International Series in Operations Research and Management Science, Springer, 166: 687-713.

[145] Yamashita M, Fujisawa K \& Kojima M. 2003. Implementation and evaluation of sdpa 6.0 (semidefinite programming algorithm 6.0). Optimization Methods and Software, 18(4): 491-505.

[146] ZHAO Q, KARISCH SE, RENDL F \& WolKowicz H. 1998. Semidefinite programming relaxations for the quadratic assignment problem. Journal of Combinatorial Optimization, 2: 71-109.

[147] ZhaO X, Sun D \& Toh K. 2010. A newton-cg augmented lagrangian method for semidefinite programming. SIAM Journal on Optimization, 20(4): 1737-1765. 